\title{
Fatores associados à qualidade de vida de pacientes
} em terapia renal substitutiva no Brasil

\author{
Factors associated with quality of life \\ in patients in renal replacement therapy in Brazil
}

Juliana Alvares ${ }^{1}$

Alessandra $\mathrm{M}$ aciel Almeida ${ }^{1}$

Daniele Araujo Campos Szuster ${ }^{2}$

I sabel Cristina Gomes ${ }^{3}$

Eli I ola Gurgel Andrade²

Francisco deAssis Acurcio ${ }^{1}$

M ariângela Leal Cherchiglia²

${ }^{1}$ Departamento deFarmácia Social, Universidade Federal deM inas Gerais. R. Silvianopolis 425/302 Bairro Santa Tereza. 31010410 Belo H orizonte M G. jualvares@gmail.com

${ }^{2}$ Departamento de M edicina Preventiva e Social, Universidade Federal de Minas Gerais.

${ }^{3}$ Departamento de

Estatística, Universidade

Federal de M inas Gerais.
Abstract End-stage renal disease (ESRD) is a serious public health problem. The study of the impact of renal replacement therapy (RRT) in quality of life $(\mathrm{Q} O \mathrm{~L})$ has become increasingly important. The aim of this study was to evaluate the QoL of patients on RRT and associated factors. 3036 patients on RRT in Brazil were interviewed in relation to socioeconomic, demographic, clinical and QoL aspects. Patients were randomly selected after a cluster sampling process on two levels: health services and patients. Q oL was measured by Eq5D. The instrument allows the indirect measurement of $\mathrm{QoL}$ and utility calculation, in addition to the direct measurement of Q oL by a visual analog scale (VAS). It was observed that transplant patients have better QoL and that the most prejudicial aspects are pain/ discomfort and anxiety/depression. Themain factors associated with Q $\mathrm{OL}$ are age, female gender, variables associated with the clinical condition of the patient such as the need for hospitalization and the presence of comorbidities, social class and variables associated with the health service use. The correlation between VAS and utility calculated was moderate and the $5 \mathrm{Eq5D}$ questions explain $43 \%$ of the variability of VAS. The calculated utility can be used in cost-utility analysis. Key words Dialysis, Renal transplant, Eq5D, Quality of life
Resumo A doença renal crônica terminal constitui grave problema de saúde pública. 0 estudo do impacto dasterapias renaissubstitutivas (TRS) na qualidade de vida (QV) tem se tornado cada vez mais importante. 0 objetivo deste estudo foi avaliar a QV de pacientes em TRS e os fatores a ela associados. Foram entrevistados 3036 pacientes em TRS no Brasil com relação a aspectos socioeconômicos, demográficos, clínicos e QV. Os pacientesforam selecionados de forma aleatória após processo de amostragem por conglomerado em dois níveis: serviços de saúde e pacientes. A QV foi medida pelo Eq5D. 0 instrumento permitea me dida indireta da QV eo cál culo da utilidade, além da medida direta da QV pela escala visual analógica (EVA). 0 bservou-sequepacientestransplantados possuem melhor QV eque os aspectos mais comprometidos são dor/desconforto e ansiedade/ depressão. Os principais fatores associados à QV são idade, sexo feminino, variáveis associadas à condição clínica do paciente, como necessidade de internação e presença de comorbidades, a classe social e variáveis associadas ao serviço de saúde utilizado. A correlação entre EVA e a utilidade calculada foi moderada e as 5 questões do Eq5D explicam $43 \%$ da variabilidade da EVA. A utilidade calculada pode ser utilizada em avaliações de custo-utilidade.

Palavras-chave Diálises, Transplante, Eq5D, Qualidadedevida 
Introdução

Em todo o mundo, incluindo países em desenvolvimento, a prevalência da doença renal crônica terminal (DRCT) tem aumentado consideravelmente nos últimos anos. As melhorias tecnológicas, especialmente as relacionadas às Terapias Renais Substitutivas (TRS) - hemodiálise, diálise peritoneal e transplante renal -, têm possibilitado maior sobrevida aos pacientes, mastambém maior chance de permanecerem com algumas incapacidades funcionais. Além disso, há ainda o desgaste emocional provocado pela doença que gera significativo impacto sobre a qualidade de vida (QV).

A QV tornou-se um importante indicador de resultados para a DRCT nas últimas duas dé cadas etem sido utilizada como preditor de agravos em saúde e de mortalidade, bem como em análises econômicas de intervenções em saúde, subsidiando a tomada de decisões. Para tanto, torna-se necessário que a QV seja quantificada aplicando-se o conceito de preferência, com base na teoria da decisão sob incerteza, pela qual se entende que os indivíduos têm preferências por diferentes estados de saúde ${ }^{1}$.

As preferências por estados de saúde podem ser mensuradas utilizando-se medidas diretas (escal a visual analógica, time trade- off estandard gamble), que empregam técnicas de valoração e escolhas, ou utilizando-se medidas indiretas (Eq5D, SF-6D, HUI), que empregam questionários desenvolvidos a partir das técnicas diretas ${ }^{2,3}$.

Vários estudos mostram que a QV de pacientes em TRS é comprometida e que pacientes transplantados tem melhores índices de QV que pacientes em hemodiálise e diálise peritoneal. M ostram ainda que fatores socioeconômicos, demográficos e clínicos exercem impacto considerável sobre a QV. A maior parte desses estudos usa como instrumento de medida o SF-36 - um instrumento genérico, composto por 36 itens que medem a QV em oito dimensões: dor, capacidade funcional, aspectos físicos, aspectos sociais, aspectos emocionais, saúde mental, vitalidade e estado geral de saúde. 0 instrumento permite a construção de duas medidas sumarizadas: os componentes físico e mental do SF-364,5.

O uso do Eq5D, apesar de pouco frequente em pacientes em TRS ${ }^{4,6}$, apresenta muitas vantagens: é de fácil manuseio, foi traduzido e validado para muitas línguas, incluindo o português, requer pouco tempo para ser respondido, uma vez que écomposto por apenas 5 questões e uma escala visual analógica (EVA). Além disso, o ins- trumento permite a comparação da QV medida em pacientes individualmente com valores pre ditos derivados de uma amostra populacional, confrontando duas diferentes perspectivas para o mesmo estado de saúde. Por fim, o escore global de QV é um resultado numérico, que pode ser utilizado como medida de utilidade e incorporado em análises econômicas' ${ }^{7,8}$. Utilidade é definida como o número que representa as pre ferências dos indivíduos em relação a resultados, podendo ser compreendido, no âmbito da saúde, como o bem-estar subjetivo ou percepção individual dos níveis de satisfação obtidos pelos pacientes ao consumir cuidados de saúde ${ }^{9}$

O objetivo deste estudo foi avaliar a QV de pacientes em TRS no Brasil, utilizando como instrumento de medida o Eq5D, e verificar sua associação com características socioeconômicas, demográficas, clínicas e relacionadas aos serviços de saúde. A identificação dos fatores associados à QV pode subsidiar intervenções sobre aqueles passíveis de modificação e, com isso, melhorar a QV de pessoas com DRCT.

\section{Material eM étodos}

Realizou-se um estudo observacional de corte transversal para avaliar a QV de pacientes em TRS no Brasil e sua relação com modalidade de tratamento, condições socioeconômicas edemográficas, efatores relacionados à condição clínica dos pacientes eaos serviços de saúde. Os participantes foram recrutados em unidades de diálise e centros transplantadores registrados na Socie dade Brasileira de N efrologia. Os critérios de inclusão no estudo foram: ter mais de 18 anos de idade, ter diagnóstico deDRCT eestar em diálise há pelo menos 3 meses ou ter realizado um transplante renal há pelo menos 6 meses. 0 termo de consentimento foi assinado por todos os pacientes incluídos no estudo. Foram excluídos os pacientes que fizeram múltiplos transplantes.

Um questionário estruturado contendo questões socioeconômicas, demográficas, clínicas ede qualidade de vida (Eq5D) foi aplicado aos pacientes por estudantes da área da saúde e por profissionais de saúde dos centros transplantadores. Os entrevistadores foram intensivamentetreinados em pesquisa, métodos de entrevistas e sobreo uso e proposta do Eq5D. Os pacientes rece biam uma cópia do questionário para acompanhar a entrevista durante sua realização. A decisão por utilizar a entrevista em detrimento à autoaplicação do Eq5D se deve ao fato de muitos 
pacientes apresentarem dificuldade para ler e escrever, o que prejudicaria ou mesmo impossibilitaria a autoaplicação em alguns casos.

Foi utilizada no estudo uma amostra representativa das unidades de diálise e centros transplantadores contratados pelo Sistema Ú nico de Saúde (SUS). Foi selecionada uma amostra por conglomerado em dois estágios, estratificada pelas modalidades de tratamento (hemodiálise, diálise peritoneal e transplante renal). No primeiro estágio foram amostradas unidades de diálise e centros transplantadores. No segundo foram selecionados pacientes em tratamento nas unidades de diálisee centros transplantadores amostrados no primeiro estágio.

O tamanho da amostra foi calculado com o propósito de permitir a comparação da QV entre pacientes em diferentes modalidades detratamento. Como o número de pacientes em cada uma é bastantediferente, ocorreu o mesmo com o quantitativo das amostras. Cada amostra é representativa da modalidade de tratamento respectiva.

A partir de dados obtidos na revisão de literatura, três parâmetros principais foram utilizados para o cálculo da amostra: nível de significância de $5 \%$, poder do teste de $80 \%$ euma diferença mínima entre duas modalidades de 5 pontos na EVA. Para hemodiálise (HD) e diálise peritoneal (DP) foi considerada uma correlação intraclasse de 0,15 (medida do grau de dependência das observações dentro de um grupo), enquanto que para o transplante renal (TR), utilizou-se um coeficiente de correlação intraclasse de 0,10. A seleção aleatória dos pacientes foi feita utilizando os registros de pacientes das unidades de diálise e centros transplantadores.

A Tabela 1 mostra a distribuição do número total de unidades de diálise e centros transplantadores no Brasil bem como o número de pacientes amostrados por modalidade de tratamento.

Para a avaliação da QV foi utilizado o instrumento denominado EuroQ ol (Eq-5D) na versão em português ${ }^{10}$. Trata-se de um questionário geral de qualidade de vida que permite gerar um escore único, necessário para a obtenção da medida de utilidade. 0 instrumento é dividido em duas partes. A primeira é composta por um sistema descritivo contendo cinco dimensões de saúde (mobilidade, cuidado pessoal, atividades cotidianas, dor/mal estar eansiedade/depressão). Cada dimensão apresenta três níveis de gravidade (sem problema, algum problema ou problema moderado e problemas mais graves). Essa etapa do instrumento permite que o indivíduo descreva, no momento da entrevista, a gravidade do estado desaúdeem cada dimensão. A combinação dos três diferentes níveis de respostas para cada um dos cinco itens definem 243 possíveis estados de saúde. A administração do Eq5D a uma amostra da população argentina ${ }^{11}$ permitiu a definição de valores para cada um dos estados de saúde (utilidade). Uma vez que no Brasil não foi realizado estudo para a definição desses valores, serão utilizados neste trabalho os encontrados para a população argentina como ponderação para o cálculo da utilidade, refletindo as preferências da população geral para esses estados de saúde. A segunda partedo instrumento consistedeuma EVA, que vai de 0 (pior estado de saúde imaginável) a 100 (melhor estado de saúde imaginável). Durante a entrevista, o paciente marca o ponto na linha vertical quemelhor reflita a valoração do seu estado geral de saúde.

0 instrumento $\mathrm{Eq}-5 \mathrm{D}$ gera dois resultados de QV: uma medida direta, feita pela EVA, euma indireta, feita pelas 5 questões e posterior cálculo dautilidade.

As variáveis usadas para explicar as variações na qualidade de vida foram socioeconômicas (classe econômica, ocupação laboral) e demográficas (sexo, idade, estado marital). Foram utilizadas as variáveis relacionadas à modalidade de tratamento de TRS, tempo em tratamento e unidade de diálise. Variáveis clínicas também

Tabela 1. Distribuição das unidades de diálise/transplantes e pacientes no universo e amostra, por modalidade de tratamento, Brasil, 2007.

\begin{tabular}{lcccccc}
\hline \multirow{2}{*}{$\begin{array}{c}\text { Modalidade de } \\
\text { tratamento }\end{array}$} & \multicolumn{2}{c}{ Universo } & & \multicolumn{3}{c}{ Amostra } \\
\cline { 2 - 3 } \cline { 5 - 7 } \cline { 5 - 7 } DP & Unidades & Pacientes & & Unidades Pacientes & M édia de Pacientes/Unidade \\
\hline HD & 421 & 8.039 & & 54 & 788 & 15 \\
TR & 610 & 93.582 & & 81 & 1.621 & 20 \\
\hline
\end{tabular}

Fonte: Sociedade Brasileira de N efrologia, 2008 [ 12]. A breviações usadas: HD: hemodiálise; DP: diálise peritoneal; TR: transplanterenal. 
foram avaliadas, tais como comorbidades, ne cessidade de consultas extras e internações no último ano. A classificação socioeconômica foi feita de acordo com os critérios da Associação Brasileira de Institutos de Pesquisa (ABIP), que divide a população em cinco grupos: A (melhor situação), B, C, D eE (pior situação). Esteíndice foi construído usando os dados da amostra estudada quanto à escolaridade do chefe da família e à posse de bens de consumo doméstico duráveis (automóvel, televisão, vídeo-casssete ou DVD, máquina de lavar roupas, aspirador de pó, geladeira, freezer), além de característica da moradia (número de banheiros).

Os pacientes foram questionados sobre a presença das comorbidades: hipertensão, diabe tes, doença cardíaca, câncer, depressão, doença pulmonar, cirrose, artrite, HIV, doença óssea, AVC, trombose, úlcera e hepatite. Essas são doenças que têm grande potencial de impacto sobre a qualidade de vida dos pacientes. Como a análise de comorbidades isoladas mostrou fraca relação com a qualidade de vida, nosso objetivo foi encontrar um conjunto decomorbidadesque, juntas, impactam sobre a qualidade de vida dos pacientes. 0 uso de índices de comorbidades já existentes não foi possível, pois algumas doenças por eles utilizadas não foram incluídas em nosso questionário. Assim, as comorbidades referidas pelo paciente foram agrupadas em um índice de comorbidades (variável contínua, com maior índice representando maior número de comorbidades). Um modelo logístico unidimensional com dois parâmetros da Teoria de Resposta ao Item (TRI) foi utilizado para gerar o índice, dada a sua habilidade para incorporar diferenças na discriminação e gravidade de cada comorbidade presente no instrumento de pesquisa ${ }^{12}$.

A análise descritiva dos dados incluiu distribuições de frequência, médias e variâncias. Foram feitas comparações entre as modalidades de tratamento com relação à QV medida de forma direta (EVA) e indireta (cálculo de utilidade). A análise de variância (ANOVA) foi utilizada para verificar diferenças entre as modalidades e, para comparações múltiplas, o pós-teste Tukey foi utilizado. A correlação entre a QV medida de forma direta e indireta foi avaliada pelo teste de correlação de Pearson. Foi feita análise de regressão line ar utilizado a EVA como variável resposta e as dimensões do Eq-5D como variáveis explicativas.

A associação entre a QV - medida de forma direta (EVA) e indireta (cálculo de utilidade) - e variáveis explicativas, incluindo a modalidade de tratamento, foi verificada por análise univariada e multivariada usando um modelo de regressão multinível. Nestemodelo, aunidadedesaúde(unidades de diálise e centros transplantadores) foi avaliada como uma variável de nível de grupo.

$\mathrm{Na}$ análise multinível, todas as variáveis independentes foram incluídas no modelo e utilizou-se o método de deleção de variáveis até a obtenção do modelo final. Os modelos inicial e final foram comparados usando o teste da razão de verossimilhança. A adequação do modelo foi avaliada usando a análise de resíduos. Utilizouse significância estatística de 5\%.

\section{Resultados}

Foram entrevistados 3.036 pacientes. Destes, 1.621 $(53,4 \%$ ) estavam em HD, 788 ( $26 \%$ ) em DP e627 $(20,6 \%)$ em TR. A maior parte era do sexo masculino (55\%) ea idade média era de 49,6 anos. A maioria dos pacientes não tinha ocupação laboral $(85 \%)$ e pertenciam às classes econômicas $C$ (43\%) ou D eE (31\%). Grande parte dos pacientes iniciou a TRS em HD (75\%) e possuem, em média, 5 anos de tratamento.

No ano anterior a entrevista, $48 \%$ dos pacientes precisaram ser internados e $12 \%$ relataram consulta médica extra, não programada. Entre os entrevistados, $85 \%$ eram hipertensos, $26 \%$ diabéticos, $23 \%$ relataram doenças cardíacas e $20 \%$ disseram possuir diagnóstico de depressão. A descrição detal hada das características socioeconômicas e demográficas, estratificadas por modalidade de tratamento, encontra-se disponível em Alvares et al. ${ }^{13}$.

As dimensões do Eq5D mostram que pacientes em HD, DP e TR apresentam diferenças em suas respostas (Tabela 2). Pacientes em DP mostram os piores resultados em todas as dimensões, especialmente na que diz respeito às atividades usuais.

De modo geral, os pacientes apresentam os maiores prejuízos nas dimensões dor/desconforto, ansiedade/depressão e atividades usuais.

A AN OVA eo testeTukey para múltiplas comparações mostraram diferença estatisticamente significativa entre as três modalidades com relação à QV medida de forma indireta (utilidade). No entanto, quando os mesmos testes foram aplicados à QV medida pela EVA, HD e DP não apresentaram diferenças significativas.

A QV medida de forma indireta, calculada com base nas preferências da população geral pelo Eq5D (utilidade), mostra melhores resultados que a QV medida de forma direta pela EVA. 


\begin{tabular}{|c|c|c|c|c|}
\hline Dimensões do Eq5Da & HD & DP & TR & Total \\
\hline \multicolumn{5}{|l|}{ M obilidade } \\
\hline 1 & $1043(64,3)$ & $355(45,1)$ & $483(77,0)$ & $1884(62,1)$ \\
\hline 2 & $543(33,5)$ & $416(52,8)$ & $143(22,8)$ & $1102(36,3)$ \\
\hline 3 & $32(2,2)$ & $17(2,1)$ & $1(0,2)$ & $50(1,6)$ \\
\hline \multicolumn{5}{|l|}{ Autocuidado } \\
\hline 1 & $1432(88,3)$ & $618(78,4)$ & $594(94,7)$ & $2644(87,1)$ \\
\hline 2 & $150(9,3)$ & $125(15,9)$ & $33(5,3)$ & $308(10,1)$ \\
\hline 3 & $39(2,4)$ & $45(5,7)$ & $0(0,0)$ & $84(2,8)$ \\
\hline \multicolumn{5}{|l|}{ Atividades usuais } \\
\hline 1 & $1006(62,1)$ & $409(51,9)$ & $485(77,4)$ & $1900(62,6)$ \\
\hline 2 & $490(30,2)$ & $281(35,7)$ & $126(20,1)$ & $897(29,5)$ \\
\hline 3 & $125(7,7)$ & $98(12,4)$ & $16(2,6)$ & $239(7,9)$ \\
\hline \multicolumn{5}{|l|}{ Dor / desconforto } \\
\hline 1 & $561(34,6)$ & $283(35,9)$ & $325(51,8)$ & $1169(38,5)$ \\
\hline 2 & $891(55,0)$ & $414(52,5)$ & $273(43,5)$ & $1578(52,0)$ \\
\hline 3 & $169(10,4)$ & $91(11,5)$ & $29(4,6)$ & $289(9,5)$ \\
\hline \multicolumn{5}{|l|}{ Ansiedade / depressão } \\
\hline 1 & $884(54,5)$ & $416(52,8)$ & $370(59,0)$ & $1670(55,0)$ \\
\hline 2 & $560(34,5)$ & $306(38,8)$ & $226(36,0)$ & $1092(36,0)$ \\
\hline 3 & $177(10,9)$ & $66(8,4)$ & $31(4,9)$ & $274(9,0)$ \\
\hline QV - medida indireta (utilidade) ${ }^{b, d}$ & $0,73 \pm 0,24$ & $0,69 \pm 0,25$ & $0,84 \pm 0,18$ & $0,74 \pm 0,24$ \\
\hline QV - medida direta (EVA) $)^{c, e}$ & $68,27 \pm 23,01$ & $67,29 \pm 22,37$ & $80,13 \pm 17,60$ & $70,46 \pm 22,34$ \\
\hline
\end{tabular}

a. 1 = nenhum problema, 2 = algum problema, 3 = problema extremo. b. Computada a partir das cinco dimensões do Eq-5D. c. M edida pela escala visual analógica. $d$. Valor- $p<0,001$ nas comparações entre HD eDP, HD eTR, DP eTR. e. Valor- $p<0,001$ nas comparações entreHD eTR, e entre DP eTR; valor- $p=0,557$ na comparação entre HD e DP. Abreviações usadas: QV: qualidade devida; HD: hemodiálise; DP: diálise peritoneal; TR: transplanterenal.

A correlação entreas medidas deQV foi de 0,386, considerada moderada.

A análise de regressão linear, utilizando a EVA como resposta e as dimensões do Eq5D como variáveis independentes, mostra que estas últimas explicam 43\% da variabilidade da EVA.

As análises de regressão hierárquica para a QV medida de forma direta (EVA) eindireta (utilidade) mostraram resultados semelhantes. Em ambas as análises, observa-se que os pacientes em TR possuem melhor QD que os em HD eDP. Quanto menor a idade, melhor a QV. Pacientes do sexo masculino, de classe social A, B ou C (em comparação com D e E), acompanhados em unidade de saúde de caráter privado, apresentam melhores índices de QV. Com relação aos aspectos clínicos, observa-se que apresentam melhor QV pacientes que não necessitaram de consultas extras e de internações e que possuem menor pontuação no índice de comorbidades. $\mathrm{N}$ a análise feita para a QV medida de forma indireta pelo Eq5D (utilidade), manteve-se no modelo final as variáveis ocupação laboral e escolaridade, enquanto no modelo para a EVA o estado marital permaneceu no modelo final. Os dados encontram-se na Tabela 3.

\section{Discussão}

A QV se mostrou substancialmente reduzida em ambas modalidades de diálise (HD e DP), com níveis similares aos observados em outros estudos $^{7}$. De W it et al. ${ }^{6}$ encontraram valores mais elevados para pacientes em DP. Os resultados obtidos naqueles em TR se aproximam dos observados na população geral.

Liem et al. ${ }^{14}$, em meta-análise, encontrou resultados de utilidade semel hantes para pacientes em TR, mas menores para pacientes em HD eDP. Tajima et al. ${ }^{15}$, em estudo desenvolvido no Japão, encontrou resultado superior ao deste trabalho para pacientes com DRCT.

Observou-se que os escores deQDV medidos de forma indireta (utilidade) são maiores que os medidos pela EVA. Essa diferença entre os instrumentos pode ser explicada pelo mecanismo psíquico de enfrentamento da doença, que faz com 
Tabela 3. Análise de regressão hierárquica de fatores associados à QV medida de forma direta (EVA) e indireta (utilidade) de pacientes em TRS no Brasil, 2007.

\begin{tabular}{|c|c|c|c|c|c|c|}
\hline \multirow[b]{2}{*}{ Variáveis } & \multicolumn{3}{|c|}{ Utilidade } & \multicolumn{3}{|c|}{ EVA } \\
\hline & Coeficiente & $\begin{array}{l}\text { Desvio } \\
\text { padrão }\end{array}$ & $p$-valor & Coeficiente & $\begin{array}{l}\text { Desvio } \\
\text { padrão }\end{array}$ & $p$-valor \\
\hline \multicolumn{7}{|l|}{ Parte fixa } \\
\hline$D P^{*}$ & $-0,004$ & 0,010 & 0,83 & $-0,044$ & 1,028 & 0,64 \\
\hline $\mathrm{TR}^{*}$ & 0,096 & 0,014 & $<0,001$ & 11,345 & 1,435 & $<0,001$ \\
\hline Idade & $-0,001$ & 0,000 & $<0,001$ & $-0,082$ & 0,030 & $<0,001$ \\
\hline Gênero (masculino) & 0,042 & 0,008 & $<0,001$ & 4,418 & 0,844 & $<0,001$ \\
\hline Internação (não) & 0,052 & 0,008 & $<0,001$ & 2,645 & 0,834 & $<0,001$ \\
\hline Consulta extra (não) & 0,023 & 0,009 & 0,015 & 2,354 & 0,853 & 0,002 \\
\hline Índice de comorbidade & $-0,106$ & 0,007 & $<0,001$ & $-3,139$ & 0,717 & $<0,001$ \\
\hline Classe social $A$ e $B^{* *}$ & 0,065 & 0,013 & $<0,001$ & 5,674 & 1,108 & $<0,001$ \\
\hline Classe social $C^{* *}$ & 0,039 & 0,010 & $<0,001$ & 3,489 & 0,964 & $<0,001$ \\
\hline Ocupação laboral (não) & $-0,024$ & 0,012 & 0,032 & - & - & - \\
\hline Estado marital (casado) & - & - & - & 2,504 & 0,851 & $<0,001$ \\
\hline Escolaridade (> 8 anos) & 0,025 & 0,010 & 0,011 & - & - & - \\
\hline Unidade de saúde privada** & 0,047 & 0,016 & $<0,001$ & 4,242 & 1,682 & 0,003 \\
\hline Unidade de saúde filantrópica** & 0,040 & 0,018 & 0,052 & 3,335 & 1,813 & 0,074 \\
\hline Parte randômica & \multicolumn{6}{|c|}{ Unidades de diálise/transplantes } \\
\hline$\sigma^{2}$ & \multicolumn{3}{|c|}{0,0007} & \multicolumn{3}{|c|}{8,799} \\
\hline$\sigma^{2}$ Residual & \multicolumn{3}{|c|}{0,044} & \multicolumn{3}{|c|}{435,80} \\
\hline $\mathrm{CCl}$ & \multicolumn{3}{|c|}{0,10} & \multicolumn{3}{|c|}{0,09} \\
\hline
\end{tabular}

* Categoria de referência: H emodiálise. ** Categoria de referência: Classe social D eE. *** Categoria de referência: H ospital público. Abreviações usadas: HD: hemodiálise; DP: diálise peritoneal; TR: transplanterenal; CCI: coeficiente de correlação intraclasse; EVA: escala visual analógica; QV: qualidade de vida

que os pacientes aprendam, gradualmente, a se adaptar à sua nova situação e a aceitar algumas limitações - tal fato se reflete mais nos métodos de medida indireta que nos de medida direta. Alguns estudos mostram que, a despeito de várias limitaçõesimpostaspela doença, pacientes em TRS vivem com QV relativamente satisfatória ${ }^{6}$. M ittal et al. ${ }^{16}$ demonstraram que, após o primeiro mês de diálise, há um aumento na qualidade de vida mental dos pacientes. Outros estudos têm mostrado uma diminuição na qualidade de vida física ao longo do tempo, mas nenhuma alteração significativa na qualidade de vida mental ${ }^{16,17}$. Neste trabal ho o tempo em tratamento não se associou de forma significativa à QV.

As variáveis associadas de forma independente com a QV - medida deforma direta eindireta - são semelhantes. A modalidade de tratamento TR compõe os modelos finais de regressão hierárquica para as duas medidas, mostrando que pacientes transplantados apresentam melhor nível de QV. Liem et al. ${ }^{14}$ e Cameron et al. ${ }^{18}$, em revisões sistemáticas e meta-análises de
QV em pacientes em TRS, encontraram resultados semelhantes. A discussão de queisso se daria pelo fato de pacientes transplantados serem mais jovens não se aplica a este trabalho, visto que o efeito da idade foi controlado pela análise multivariada. Quanto ao estado clínico dos pacientes, observa-se que, quanto maior a pontuação no índice de comorbidades, pior a QV; e os que necessitaram de internações ou consultas extras também demonstraram pior QV. Aqueles nas classes sociais A, B eC (em comparação com D e E) têm QV melhor. Osatendidos em unidades de saúde privadas, em relação às públicas, tem meIhor QV. Estes resultados são esperados e concordam com a literatura.

As diferenças entre os dois modelos está no fato de que, na análise da medida indireta de QV (utilidade), o estado marital dos pacientes não permaneceu no modelo final, enquanto no modelo da medida direta (EVA), não permaneceram as variáveis ocupação laboral ea escolaridade. $\mathrm{Na}$ literatura épossível observar que, em estudosque utilizam a mesma população e instrumentos de 
medidas diferentes, ocorrem al gumas diferenças nos modelos multivariados finais. A explicação para essas diferenças pode estar no fato de que as medidas direta e indireta refletem diferentes aspectos do conceito de $\mathrm{QV}$, um enfoca o perfil de saúde em relação a determinados domínios da QV e o outro explicita o julgamento individual sobre o estado de saúde em determinado momento. A principal implicação desse achado éque as medidas direta e indireta podem levar a diferentes conclusões sobre a QV dos pacientes ${ }^{6,7}$.

A correlação entrea EVA e o Eq5D foi moderada e concorda com os resultados encontrados na literatura $0^{6,7,19}$. Várias razões podem explicar esse achado: primeiro, pessoas diferentes atribuem valores diversos para o mesmo estado de saúde e, consequentemente, têm preferências diferentes para esses estados de saúde. Em segundo lugar, na medida indireta (utilidade) os valores são derivados de um estudo da população geral argentina, enquanto os valores medidos diretamente pela EVA refletem a opinião do paciente brasileiro no momento da pesquisa. Por último, as 5 dimensões do Eq5D são avaliadas com uma escala de três níveis (nenhum problema, algum problema e problema grave), o que pode forçar uma resposta "algum problema". Como poucos pacientes endossam a resposta "problema grave" e como alguma limitação está frequentemente presente em pacientes em TRS, a resposta do meio acaba sendo a melhor opção. Badia et al..$^{20}$, comparando os resultados obtidos com as cinco dimensões e a EVA, mostraram resultados consistentes com esta hipótese.

Entretanto, essa relativa limitação do Eq5D pode ser vista como sua grande vantagem: 0 instrumento consegue derivar as preferências dos pacientes e da população geral ao mesmo tempo e, dessa forma, as duas perspectivas podem ser incorporadas em estudos que verificam $0 \mathrm{im}$ pacto de diagnósticos ou alternativas terapêuticas, como as análises econômicas9,20.

A medidaindireta deQV (utilidade), feita pelo Eq5D, mede aqualidadedevida por meio decinco componentes, que refletem as dimensões física, funcional e mental da QV. Juntas, essas dimensões explicam $43 \%$ da variabilidade da QV medida de forma direta pela EVA e todas as dimensões permanecem no modelo final de regressão. As dimensões que obtiveram os maiores coeficientes na análise de regressão linear foram dor/desconforto e ansiedade/depressão. Os resultados encontrados concordam com os de
Wasserfallen et al. ${ }^{7}$, únicos autores a também fazerem esse tipo de análise, e que discutem a necessidade de maiores investigações no sentido de avaliar a relação entre as medidas direta e indireta de QV e o que de fato elas se propõe a medir.

Algumas limitações deste estudo devem ser destacadas. Em primeiro lugar, trata-se de um estudo de corte transversal e, por isso, não é possível determinar com exatidão o quanto o tratamento é o responsável pela perda da QV. Em segundo lugar, ainda não foram feitos estudos que possibilitem o cálculo da utilidade para a população brasileira e, por isso, foram utilizados os parâmetros da Argentina. 0 uso dos escores argentinos pode não ser o mais adequado para a população do Brasil. Além disso, variáveis clínico-laboratoriais, que podem interferir nos resultados de QV, como al bumina e creatinina, não foram avaliadas.

Como no Brasil a quase totalidade das TRS são custeadas pelo SU S ea DRCT representa um alto impacto financeiro para o sistema público de saúde, é importante ressaltar que este é o primeiro trabalho a medir a qualidade de vida e estimar a utilidade de pacientes em TRS utilizando amostra significativa das unidades de diálisee centrostransplantadores no Brasil eservirácomo base para futuros trabalhos na área da economia da saúde, em estudos de custo-utilidade ${ }^{21}$.

0 que se sabe no momento é que, embora alguns pacientes tenham uma preferência médica ou social por alguma modalidade de tratamento, uma grande proporção dos que iniciam a TRS pode ser tratada com sucesso com qualquer uma ${ }^{22}$. A escolha é uma decisão complexa, em que fatores clínicos, não clínicos e, em especial, a qualidade de vida, devem ser levados em consideração $0^{23}$.

Por fim, éimportanteressaltar quetransplante e diálise não podem ser analisados separadamente. Um programa de transplante de sucesso requer uma terapia dialítica eficiente, que dá ao paciente suporte para a cirurgia e o recebe em caso de perda do órgão transplantado. A dependência mútua também se aplica às diferentes modalidades de diálise: pacientes quetêm ou desenvolvem contraindicações para determinada modalidade precisam ter acesso a outra modalidade/opção terapêutica ${ }^{24}$. É importante que 0 custo-efetividade da TRS seja avaliado em um nível agregado, tendo como foco do tratamento o bem-estar ea maior ampliação possível da longevidade do paciente. 


\section{Colaboradores}

J Alvares, AM Almeida, DAC Szuster, IC Gomes, EIG Andrade, FA Acurcio e M L Cherchiglia participaram igualmente de todas as etapas de elaboração do artigo.

\section{Referências}

1. Prieto L, Sacristán JA. Problems and solutions in calculating quality-adjusted life years (QALY's). Health Qual Life outcomes 2003; 1:80.

2. Guyatt GH. A taxonomy of health status instruments. J Rheumatol 1995; 22(6):1188-1190.

3. Higginson IJ, Carr AJ. Using quality of life measures in the clinical setting. BMJ 2005; 322(7297): 1297-1300.

4. Glover C, Banks P, Carson A, Martin CR, Duffy T. Understanding and assessing the impact of end stage renal disease on quality of life. Patient $2011_{\text {; }}$ 4(1):19-30.

5. Ware JE, Kosinski M, Bjorner JB, Turner-Bowker DM, Gandek B, M aruish ME. SF-36v2 ${ }^{\circledR} \mathrm{H}$ ealth Survey: A primer for healthcare providers. Lincoln, RI: QualityM etric Incorporated; 2008.

6. De Wit GA, M erkus MP, Krediet RT, Charro FT. $\mathrm{H}$ ealth profiles and health preferences of dialysis patients. Nephrol Dial Transplant 2002; 17(1):86-92.

7. Wasserfallen JB, Halabi, G, Saudan P, Perneger $T$, Feldman HI, Martin PY, Wauters JP. Quality of life on chronic dialysis: comparison between haemodialysis and peritoneal dialysis. Nephrol Dial Transplant 2004; 19(6):1594-1599.

8. Pinto EB, Maso I, Vilela RNR, Santos LC, OliveiraFilho J. Validation of the EuroQol quality of life questionnaire on stroke victims. Arq N europsiquiatr 2011; 69(2-B):320-323.

9. Torrance GW. M easurement of health state utilities for economic appraisal. J Health Econ 1986; 5(1):130.

10. EuroQ ol Group. EQ-5D a measure of health-related quality of life developed by the EuroQ ol group: user guide. $7^{\text {th }}$ Editon. Rotterdam: EuroQ ol Group; 2000.

11. Augustovski FA, Irazola VE, Velazquez AP, Gibbons $L, C$ raig BM. Argentine valuation of the Eq5D health states. Value Health 2009; 12(4):587-596.

12. Rizopoulos D. Itm: An R package for latent variable modeling and item response theory analyses. J Stat Soft 2006; 17(5):1-25.

13. Alvares J, Cesar CC, Acurcio FA, Andrade EIG, Cherchiglia ML. Quality of life of patients in renal re placement therapy in Brazil: comparison of treatment modalities. Qual Life Res 2012; 21(6):983-991.

14. Liem YS, Bosch JL, H unink M GM. Preference-based quality of life of patients on renal replacement therapy: a systematic review and meta-analysis. Value $\mathrm{H}$ ealth 2008; 11(4):733-741.

\section{Agradecimentos}

Ao apoio financeiro do Fundo Nacional de Saúde/M inistério da Saúde, Departamento de Ciências eTecnologia em Saúde do M inistério da Saúde/Conselho Nacional de Pesquisa - CN Pq e Fundação de Amparo à Pesquisa do Estado de M inas Gerais - FAPEMIG.

15. Tajima R, Kondo M, Kai H, Saito C, Okada M, Takahashi H, Doi M, Tsuruoka S, Yamagata K. M easure ment of health-related quality of life in patients with chronic kidney disease in Japan with Euroqol (Eq5D). Clin Exp Nephrol. 2010; 14(4):340-348.

16. Mittal SK, Ahern L, Flaster E, M aesaka JK, Fishbane $\mathrm{S}$. Self-assessed physical and mental function of haemodialysis patients. Nephrol Dial Transplant 2001; 16(7):2152-2159.

17. Baiardi F, Esposit ED, Cocechi R, Fabri A, Sturani A, Valpiani G, Fusarol M. Effects of clinical and individual variables on quality of life in chronic renal failure patients. J Nephrol 2002; 15(1):61-67.

18. Cameron JI, Whiteside C, Katz J, Devins GM. Differences in quality of life across renal replacement therapies: a meta-analytic comparison. Am J Kidney Dis 2000; 35(4):629-637.

19. Campolina AG, Bortoluzzo AB, Ferraz MB, CiconelIi RM. Validação da versão brasileira do questionário genérico de qualidade de vida short-form 6 dimensions (SF-6D Brasil). Cien Saude Colet 2011; 16(7):3103-3110.

20. Badia X, Herdman M, Schiaffino A. Determining correspondence between scores on the EuroQ ol EQ-5D 'thermometer'and a 5-point categorical rating scale. M ed Care 1999; 37(7):671-677.

21. Campolina AG, Cicconeli, RM . Qualidade de vida e medidas de utilidade: parâmetros clínicos para as tomadas de decisão em saúde. Am J Public H ealth 2006; 19(2):128-136.

22. Lee CP, Chertow GM, Zenios SA. An empiric estimate of the value of life: updating the renal dialysis cost-efectiveness standard. Value Health 2009 ; 12(1):80-87.

23. Salonen $T$, Reina $T$, O ksa $H$, Rissanen $P$, Pasternack. Alternative strategies to evaluate the cost-effectiveness of peritoneal dialysis and hemodialysis. Int U rol Nephrol 2007; 39(1):289-298.

24. De Wit GA, Ramsteijn PG, Charro FT. Economic evaluation of end stage renal disease treatment. H ealth Policy 1998; 44(3):215-232.

Artigo apresentado em 07/11/2012

Aprovado em 15/01/2013

Versão final apresentada em 23/01/2013 